\title{
The middle of the road: Perceiving intermediates
}

\author{
Ivana Bianchi ${ }^{\mathrm{a}, *}$, Roberto Burro ${ }^{\mathrm{b}}$, Stefania Torquati ${ }^{\mathrm{c}}$, Ugo Savardi ${ }^{\mathrm{b}}$ \\ a Department of Humanities, University of Macerata, Italy \\ b Department of Philosophy, Education and Psychology, University of Verona, Italy \\ ${ }^{c}$ Department of Educational Sciences, University of Macerata, Italy
}

\section{A R T I C L E I N F O}

\section{Article history:}

Received 13 July 2012

Received in revised form 8 February 2013

Accepted 20 May 2013

Available online $\mathrm{xxxx}$

\section{PsycINFO classification:}

2323 Visual Perception

2340 Cognitive Processes

Keywords:

Intermediates

Opposites

Spatial properties

Bipolar dimensions

Grounding cognition in perception

\begin{abstract}
A B S T R A C T
This article aims to study the extension and immediacy of the perception of intermediates during the observation of images showing a variation in a spatial property from one extreme (e.g. at the top of a mountain) to the opposite extreme (e.g. at the bottom of a mountain). Three experiments were carried out: rating tasks were used in studies 1 and 3 and a classification task in study 2 .

Three main results emerged. The first result (concerning extension) is that people consistently recognize some instances of a dimension as intermediates (neither $a$... nor $b$ ) rather than as one or the other opposite pole $(a, b)$. The number of these cases ranges from one to most of the experiences in between the two extremes, depending on the type of opposite considered. The second result (concerning immediacy) is that recognizing and rating intermediates did not take longer in most cases than recognizing and rating the two poles. The third result (concerning task influence) is that there were differences due to the type of task, i.e. rating and classification. The implications of these results are discussed within the framework of theories grounding cognition in perception.
\end{abstract}

(c) 2013 Elsevier B.V. All rights reserved.

\section{Introduction: intermediates and the cognitive structure of opposites}

Antonymy is one of the relationships which has been studied most frequently by linguists due to its great importance in everyday language (Cruse, 1986; Lyons, 1977; Murphy, 2003). This importance has also been acknowledged in recent approaches to the subject in the field of cognitive linguistics: "It is well-known to both lay people and researchers interested in the meaningful functioning of language that antonymy (...) as a binary contrast used in order to express opposition, is a commonplace in all kinds of communicative modalities and registers: written as well as spoken, fact as well as fiction and formal as well as informal. Antonymy is also important in the design of iconic signs, such as traffic signs, and in visual works of art of different kinds (Giora, Heruti, Metuki, \& Ofer, 2009)..." (Paradis \& Willners, 2011). Everyday behavior is regulated by this relationship, more than one might realize: "Try visiting a public lavatory without checking which is the 'gents' and which is the 'ladies'. On your way out, ignore the instructions which tell you whether to 'push' or to 'pull' the door. And once outside, pay no attention to whether traffic

\footnotetext{
* Corresponding author at: Department of Humanities, Section: Philosophy and Human Sciences, University of Macerata, Via Garibaldi, 20, 62100 Macerata, Italy. Tel.: + 390733 2584320.

E-mail address: ivana.bianchi@unimc.it (I. Bianchi).
}

lights are telling you to 'stop' or 'go'. At best, you will end up looking very foolish; at worst, you will end up dead" (Jones, 2002, p. 7). Even speakers innocent of semantic theory have robust intuitions about lexical opposites, and children rapidly catch on to the idea at an early age (Croft \& Cruse, 2004). The importance of opposition in cognition emerges in pre-linguistic phases of human development (see Casasola, 2008; Casasola, Cohen, \& Chiarello, 2003; Quinn, 2005).

This primacy and pervasiveness might be due to the fact that opposition is a relationship rooted more in perceptual than linguistic structures and a new investigation of opposites based on this hypothesis has been put forward (Bianchi \& Savardi, 2008a, 2012; Bianchi, Savardi, \& Burro, 2011; Bianchi, Savardi, \& Kubovy, 2011; Savardi, 2009). There are a number of new questions being asked. For example: is there evidence, on the level of perceptual judgments, that two contraries lay on a common underlying dimension (Bianchi, Savardi, \& Burro, 2011)? Is the range of experiences which are perceived as belonging to one pole more extensive or topologically different from the other pole (Bianchi, Savardi, \& Kubovy, 2011)? What are the transformations of a given object which are immediately recognized as generating its opposite (Bianchi \& Savardi, 2006; Bracco, Bianchi, Chiorri, Burro, \& Savardi, 2009)? And are there experiences that we perceive as 'neither a, nor b', and, if so, what is their status? This paper focuses on the last question. It deals with experiences which are in between two poles but are not perceived as gradations of one or 
the other pole, being rather recognized as neither of them - and we will refer to these throughout the paper using the term 'intermediates'. ${ }^{1}$

Informal observations provide plenty of evidence that humans perceive some properties as intermediates (neither one pole nor the opposite) and that this is common to various sense modalities. When something which is initially very close to you moves further away, for a certain range of distances it is still perceived as near (although at varying degrees). This is followed by a range of distances that are perceived as neither near nor far before the object is perceived as being far away (and progressively further with various gradations of distance). Similarly, a tone of the voice can be perceived as low, high or neither low nor high; a cup of tea as sweet, bitter or neither sweet nor bitter; a street may be downhill, uphill or neither downhill nor uphill (i.e. on a level); a pair of shoes are loose-fitting, tight or neither loose nor tight (and only if they are neither loose nor tight do we buy them). Linguistically, intermediates are in most cases expressed by a double negation (e.g. neither small nor large; neither full nor empty; neither long nor short...) or by terms which work as cover-alls for very different dimensions (e.g. medium or normal, applied to size, brightness, weight, intensity of smell and many more); only rarely are they expressed using specific terms (e.g. tepid). But these linguistics aspects are not what we are interested in here. We aim to define the range of qualitative variations leading from an extreme property to its opposite comprising one or a series of properties which are recognized by an adult observer as neither one pole nor the other. And this question has to be addressed in terms of phenomenological psychophysics (Kubovy, 2003; Kubovy \& Gepshtein, 2003).

A taxonomy of intermediates with regard to spatial properties had already been put forward in a recent work (Bianchi, Savardi, \& Kubovy, 2011). In the present paper we wondered: (a) whether we can provide support for this taxonomy - and evidence that it is perceptually based - using more traditional methods of experimental psychology than those used in the original study; (b) whether we can demonstrate that the recognition of intermediates does not take longer than the recognition of poles - and we consider this to be evidence of the fact that they are immediately perceived and not derived by means of a double exclusion process involving the two polar components - and (c) whether differences emerge when rating tasks versus classification tasks are used.

Before presenting the procedures and results of the studies carried out, two other observations are relevant to set the framework of these studies.

I) Perceiving the middle, perceiving intermediates: Even though a phenomenological psychophysical investigation of intermediates is only at the early stages, there are, on the other hand, a considerable number of psychophysical studies where bisection tasks have been used to test people's ability to find the exact middle of a line during visual or haptic exploration (Bowers \& Heilman, 1980; Brooks, Della Sala, \& Logie, 2011; Fink, Marshall, Weiss, \& Zilles, 2001; Gallace, Auvray, \& Spence, 2007; Masin, 2008; Millar \& Al-Attar, 2000; Post, O'Malley, Yeh, \& Bethel, 2006), of chromatic scales (Cavézian, Valadao, Hurwitz, Saoud, \& Danckert, 2012; Mattingley, Bradshaw, Nettleton, \& Bradshaw, 1994), or of auditory dimensions (Dufour, Touzalin, \& Candas, 2007; Ocklenburg,

\footnotetext{
${ }^{1}$ Studies on degree modifications in linguistics (e.g. Kennedy \& McNally, 2005; Paradis, 2000) are not studies on intermediates in the sense intended in this study (i.e. experiences perceived as neither $a$, nor $b$ ). They demonstrate that properties are gradable (e.g. quite large; almost at the top). This gradability is in a sense a necessary condition in order to have something in between the two extremes but this 'something in between' may be recognized as gradations of pole $a$ or gradations of pole $b$ and not necessarily as neither $a$ nor $b$, which is instead what we have been specifically investigating here (i.e. a 'third component' of dimensions, when it exists).
}

Hirnstein, Hausmann, \& Lewald, 2010; Sosa, Clarke, \& McCourt, 2011). What is often emphasized in this literature is that there are systematic biases in what healthy people perceive as being the exact middle which does not perfectly correspond to the physical middle (an extensive meta-analysis is reported in Jewell \& McCourt, 2000; McCourt \& Olafson, 1997). However, what these studies also prove is that the task is easily feasible - people can perform it even with a single exploration (Lee et al., 2004) - and the ability to bisect does not only apply to a single sense mode but to various sense modes (which is why blind people can also do it - see Cattaneo et al., 2011). In other words, there is extensive and incontrovertible evidence that the perceptual system is tuned to find the middle and any errors generally concern precision. Additional evidence of this automatic ability in animal cognition has also been found (Mannella \& Baldassarre, 2007; Tommasi \& Thinus-Blanc, 2004; Tommasi \& Vallortigara, 2000; Tommasi, Vallortigara, \& Zanforlin, 1997). Furthermore, the special status of the center in human perception has been emphasized in literature which is not strictly psychophysical (for instance Arnheim, 1988).

All this literature is relevant to the present paper since it demonstrates the sensitivity of the cognitive system when focusing on what is 'around the middle' (etymologically, inter-medius). However, while bisection tasks point to a special case of intermediates since what is being sought is the single experience (a point, a tone, a pitch, an intensity) perceived as the exact middle between two endpoints, in the ecological world the perception of intermediacy is not necessarily associated only with a single experience but may extend also to ranges of experiences. For instance, it is likely that when one looks at a climber exactly in the middle between the top and the bottom of a mountain, one perceives him or her to be in an intermediate position. However, there is a wider range of positions that the climber might occupy where we would still perceive him/her as being neither at the bottom nor at the top of the mountain.

II) Looking for invariance: Literature on situated cognition (e.g. Clancey, 1997) has emphasized that cognition is affected by context, and some perceptual studies have shown that perception is also affected, to a certain degree, by subjective variables. For example with regard to spatial properties, which are the subject of this paper, slant perception turned out to be in part affected by mood (e.g. Riener, Stefanucci, Proffitt, \& Clore, 2011) or object size by apparent grasping abilities (e.g. Linkenauger, Witt, \& Proffitt, 2011). It is obvious to us that the perception of being at the midpoint of or at the top of a mountain might change to a certain degree when ascending or descending, or might depend on how tired the climber is. However, subjective conditions are not the focus of this study - whereas context has been partially taken into consideration by applying the same dimensions to two different objects (as explained in the method section of study 1). Our main focus here, as in previous works on the phenomenological psychophysics of opposites (Bianchi, Savardi, \& Burro, 2011; Bianchi, Savardi, \& Kubovy, 2011) is to test invariance.

\section{Starting point and research questions}

According to the taxonomy identified by Bianchi, Savardi, and Kubovy (2011) - which, to avoid repetition, we will refer to in the following pages as BSK - spatial dimensions are characterized by 4 different phenomenological structures, defined by the topological and metric characteristics of pole A, intermediates, and pole B.

i) Point-No intermediates-Range (PNR): prototype completeincomplete. These dimensions are metrically characterized by strong asymmetry and a negligible intermediate region. Topologically, the shorter pole is in most cases a single experience, what in topology is called a point; in some, rarer, cases it is a restricted bounded range. The longer pole is an unbounded range; the intermediates are non-existent; 
ii) Range-Range-Range (RRR): prototype large-small. These dimensions are metrically characterized by moderate asymmetry and an intermediate region covering around $1 / 3$ of the dimension. Topologically, the shorter pole is a bounded range and the longer pole an unbounded range; the intermediates are a range;

iii) Point-Range-Point (PRP): prototype full-empty. These dimensions are metrically characterized by slight asymmetry and an extensive intermediate region covering around $2 / 3$ of the dimension. Topologically, the poles are usually points or less frequently bounded ranges; the intermediates are a range;

iv) Range-Point-Range (RPR): prototype inside-outside. These dimensions are metrically characterized by minimal asymmetry and an almost inexistent intermediate region. Topologically, the poles are symmetrically either bounded or unbounded ranges; the intermediate is a point.

These structures emerged in two experiments where adult participants (working in inter-observation groups) were asked to focus on as many real life experiences of spatial properties as they could directly experience in the surrounding environment in addition to objects/ environments not under observation at that moment in time but which they had experienced in the past and could inter-subjectively refer to.

The aim of the three studies presented in this paper was to verify whether we can support this taxonomy using more traditional methods of experimental psychology. We paid particular attention to the structure of intermediates in order to determine first, whether intermediates are a necessary component in the structure of dimensions, i.e. whether some instances of the dimension are recognized as instances neither of one pole nor of the other; second, whether the extension of this subset varies in agreement with the abovementioned taxonomy and is quite independent of the specific object to which the property is applied; third, in addition to the two former objectives which are common to all three studies, in study 1 we also aimed to verify whether the response times needed to recognize and rate intermediates are not in general slower than times needed to recognize and rate the properties of poles. If intermediates are directly perceived as easily as extreme polarized properties longer times should not be required; fourth, we aimed to understand whether the task (classification task versus rating task) impacts on the definition of the structure of dimensions and to determine which of these tasks is more in agreement with BSK taxonomy.

\section{Study 1}

The aim of this study was to verify whether the four structures described in the previous section (PRP, RPR, RRR, PNR) are upheld in a standard rating task and a standard setting (see procedure). We paid special attention to the intermediate component, with the aim of understanding whether there are some instances of a dimension (and if so how many) that are perceived as neither one pole nor the opposite pole. We also wished to establish whether the time needed to perceive and rate the presence of an intermediate property in a scene (e.g. neither far nor near) was longer than that required to perceive and rate the presence of the two polar properties (e.g. far, near). If intermediates have the same perceptual status as polar properties, a longer period of time would not be necessary to perceive and rate them.

\subsection{Method}

\subsubsection{Participants}

43 adults (aged 20-43) divided into two groups (21 in group 1, 22 in group 2).

\subsubsection{Materials}

16 series of 23 color pictures were used. The 23 images showed the same scene, but each image differed slightly in terms of the property in question, varying from an extreme state (e.g. at the top) to the opposite state (e.g. at the bottom). In between the two extremes there were constant increments through 21 intermediate stages. An example of the 23 images forming the at the top-at the bottom series is shown in Fig. 1. We decided to show 23 variations since they represented qualitatively noticeable increments which were, however, small enough for a sensitive scale according to the idea of 'phenomenological threshold' (put forward in Bianchi, Savardi, \& Kubovy, 2011). The series involved eight different contraries sampled from the list of 37 basic spatial dimensions described in the same paper. Each set of contraries was applied to two different objects/situations (Table 1).

The two objects/situations were chosen in a preliminary interobservational phase by 4 expert judges according to the criterion that the property being observed had two different perceptual instantiations in the two conditions (e.g. it applied to continuous versus discrete objects, to a random versus an ordered pattern, or to one versus several objects). We opted for this criterion in order to enhance the generalizability of the results.

\subsubsection{Procedure}

A stand alone software (programming language: Actionscript 3 for Adobe AIR runtime environments) was used for the presentation of stimuli and the collection of response times and ratings. Eight series (4 Types $\times 2$ Dimensions) of 23 images were presented to each group of participants. The variables within subject were Types (PRP, RRR, PNR, RPR), Dimensions (2 for every Type), Images (23 images forming a series, showing variations of a property from one pole to the opposite pole) and Components (pole a, intermediates, pole b), whereas the Object/scene to which each dimension was applied varied between groups (see Table 1 - each group saw only one object/scene per dimension).

Each series of 23 images was presented three times: on one occasion the participants were asked to look at the images and rate how much evidence there was of property $a$ (e.g. at the top); on another occasion they looked at and rated how much evidence there was of property $b$ (e.g. at the bottom) and on yet another occasion they observed and rated the evidence of a property which was neither $a$ nor $b$ (e.g. neither at the top nor at the bottom). The three repetitions of each series were never presented one immediately after the other, but at a distance of at least three series: after rating, for example, how much evidence there was of the property at the top in the series regarding the object 'mountain', participants were presented with three or four series of images referring to different objects and different pairs of opposites (e.g. near, neither beginning nor end, complete) and only at this point were they shown the series of images referring to the 'mountain' again, being asked this time to rate one of the other two components which had not yet been considered (i.e. at the bottom or neither at the top nor at the bottom). Within each series the 23 images were presented in random order.

A question appeared at the center of the screen (e.g. "to what extent, looking at the position of the flag with respect to the mountain, do you perceive the property at the top?"). Participants were asked to read the question carefully and, when ready, to press a key on a keyboard to make the first image appear (images were full screen). When ready to respond, participants pressed the space bar; response times were recorded and a min-max scale appeared. Participants expressed their rating by moving the cursor along the scale (ratings were automatically recoded in a numerical scale ranging from 0 to 100 and recorded). The initial question appeared again at the center of the screen and a new image randomly followed. This occurred for all 23 images in each series. After a series was finished, the question changed and a new series of images was presented.

The instructions were verbally provided at the beginning of the experiment, and the meaning of the term "intermediate" was explained 


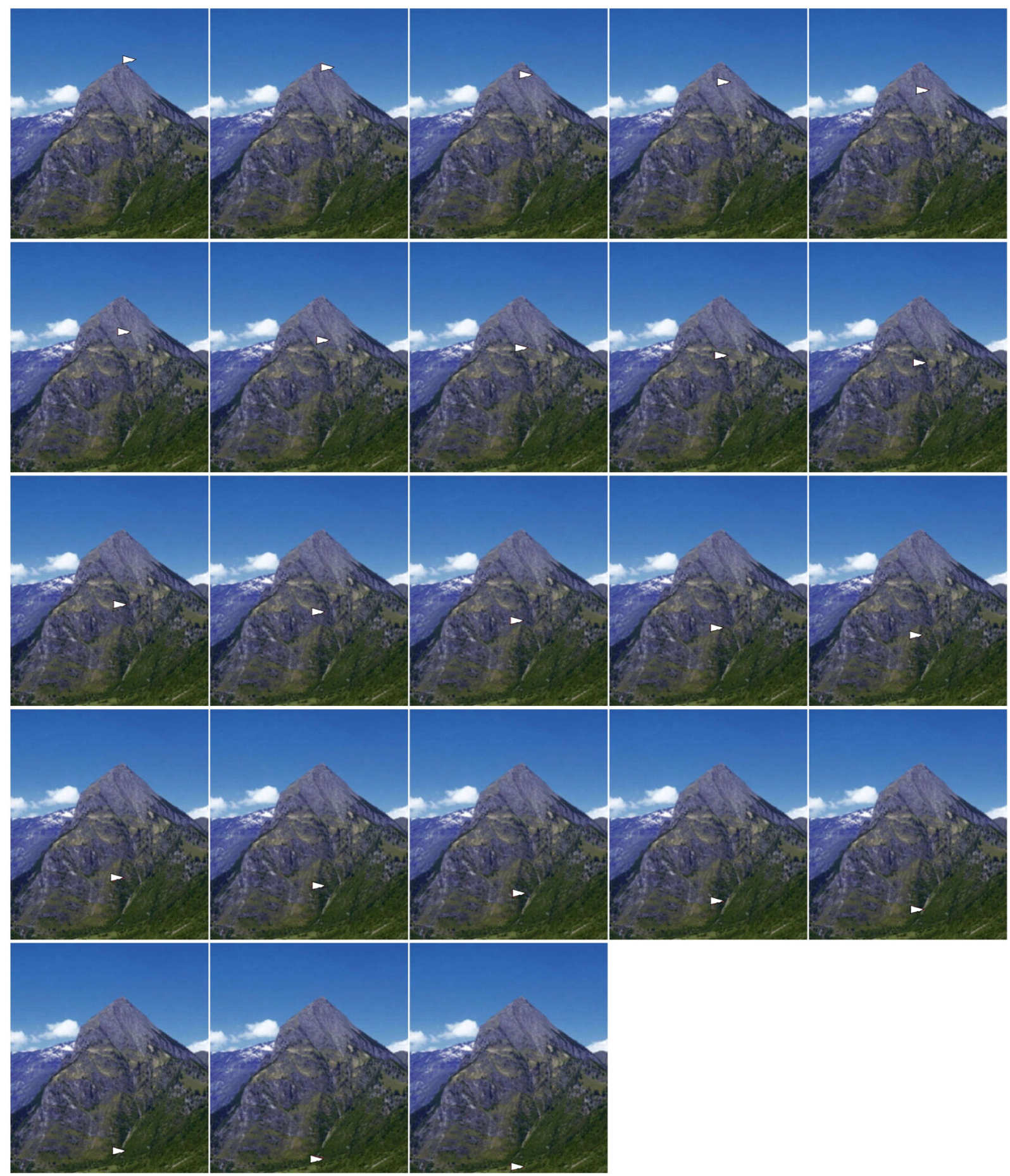

Fig. 1. The 23 stimuli used for the pair of contraries at the top-at the bottom referring to the position of a flag on a mountain.

(neither a nor $b$, not both $a$ and $b^{2}$ ). The participants were invited to rely on their own perception. It was also explained that their response time would be recorded. Since the experiment lasted on average 60 minutes,

\footnotetext{
${ }^{2}$ Phenomenally speaking, the experience of neither $a$ nor $b$ is not the same as the experience of both $a$ and $b$. For instance, a shoe that is neither loose nor tight is not both loose and tight; tea which is neither sweet nor bitter is not both sweet and bitter. Note that this holds independently of the fact that in some cases the intermediate state might in fact really have been obtained by means of a combination of two opposite properties (like, for example, when one adds cold water to hot water in order to cool it down or when one adds white color to black color to obtain gray). When we experience that the water is neither hot nor cold, we do not experience simultaneously a bit of both poles, but a new state which is neither of them.
}

participants were invited to take a short break every time the question changed (i.e. before a new series of images).

\subsection{Results}

3.2.1. Time needed to recognize and rate the intermediates and the two poles.

Response Times (RTs) provide an indication of whether recognizing the presence of intermediates and quantifying them took more time than recognizing and quantifying the presence of qualities related to the two poles. If the perception of intermediates is not immediate but 
Table 1

Spatial opposites (belonging to the four structure types) studied in the three experiments presented in the present paper, each applied to two different objects/situations.

\begin{tabular}{lll}
\hline $\begin{array}{l}\text { Type of } \\
\text { contrary }\end{array}$ & $\begin{array}{l}\text { Contrary properties } \\
\text { (Dimensions) }\end{array}$ & Applied to (objects/situations) \\
\hline PNR & $\begin{array}{l}\text { Complete-incomplete } \\
\text { Ordered-disordered }\end{array}$ & $\begin{array}{l}\text { A cake, a car park } \\
\text { A set of arrows, a set of playing cards }\end{array}$ \\
PRP & Beginning-end & A path, a dictionary \\
& At the top-at the bottom & A mountain, a ladder \\
RPR & In front of-behind & Two runners, a computer monitor \\
& Above-below & A thermometer, a mountain chalet \\
RRR & Near-far & A seat in a theater, a ball on a football \\
& Dense-sparse & pitch \\
& & A random set of paper confetti, the \\
& & teeth of a comb
\end{tabular}

a The experiment was done in Italian: the attributes "completo-incompleto" (English "complete-incomplete") used to refer to a cake and a car park are perfectly appropriate in Italian.

is secondary to the perception that something is not $a$ and at the same time is not $b$, greater response times should be associated with ratings of intermediates than with ratings of $a$ or $b$.

A first exploration of the data with a conventional linear model on RTs (ANOVA) was carried out for each dimension. The QQ-plots showed that the residuals were far from normally distributed (an example is provided in Fig. 2, diagram on the left). To check what an appropriate transformation of the raw RTs might be, we looked at Box-Cox plots (Fig. 2, central diagram), which suggested the $\log$ (RTs) transformation. Conventional linear models were then applied to $\log$ (RTs) and indeed the QQ-plots showed that the residuals were approximately normally distributed (Fig. 2, diagram on the right).

We therefore performed our final analyses on $\log (\mathrm{RTS})$, using linear mixed-effect models (REML criterion estimate). Fig. 3 shows how $\log$ (RTs) on average changed for the three components.

What was particularly interesting was to determine whether the rating of neither-nor was slower that the ratings of the poles. We tested this with Tukey multiple comparison tests on the results of the mixed-effect models (see Fig. 4). As confirmed by these tests, the rating of neither-nor was systematically slower for only 2 of the 8 dimensions (dense-sparse and beginning-end). For the other dimensions, in some cases the $\log$ (RTs) associated with intermediates were in between the $\log$ (RTs) of the poles, which means that rating intermediates was slower than rating one pole but faster than rating the other - this happened for complete-incomplete (cake and car park), in front-behind (runners), and near-far (football). In other cases ratings of intermediates were faster than those of both poles - this happened for above-below (chalet and thermometer), ordered-disordered (arrows and cards), and top-bottom (ladder and mountain). In the remaining cases, rating intermediates was slower than rating one pole but the same speed as rating the other pole - this happened with in front-behind (monitor) and near-far (theater). In conclusion, for 12 out of the 16 series analyzed, the time to rate neither-nor was shorter than the time to rate at least one pole (and in 6 of these 12 series it was shorter than both poles) confirming our hypothesis that intermediates are not harder to recognize and rate than polar properties. No systematic association was found between $\log (\mathrm{RTs})$ and types.

\subsubsection{Rating data}

The ratings for each series of figures are represented in Fig. 5, together with the regression line resulting from the application of Local regression modeling (LOESS). LOESS fits simple models to localized subsets of the data to build up a function that describes the deterministic part of the variation in the data, point by point.

Some issues emerge from these diagrams. Firstly, for some components (i.e. above, neither above nor below and below; beginning, neither end-nor beginning and end; top and even more clearly bottom) participants seemed to fall into two categories and the LOESS smoothing curves are in these cases a compromise between the two. For all other pairs there was a clear unique trend of responses. Secondly, there was a large number of zero ratings indicating a reciprocal exclusion of the properties; in other words when one was present the others were frequently not perceivable. Thirdly, the whole scale of ratings is also used for intermediates (as for the poles), which means that maximum evidence was recognized even when neithernor properties were involved. Finally, there appeared to be striking differences between the ratings of poles and intermediates belonging to different types of dimensions whereas similar patterns characterize the curves referring to the same dimension but applied to two different objects. To test this last aspect we applied 2-sample Permutation Tests to the set of values forming the regression curves related to object 1 and object 2, for each dimension. As shown in Table 2 no significant differences were found for any of the curves.

What does the shape of the curves suggest regarding the internal structure of the various types of dimensions analyzed? Let us discuss this by focusing on where the zero ratings apply. With regard to intermediate properties (neither-nor): 1) in general, zero ratings of neither-nor characterize the images at the two extremes of each series (towards stimuli 1 and 23 respectively) and this confirms that intermediates are not visible when extremely well polarized properties are shown - in other words, there are at least two instances of each dimension that are perceived as opposite polar properties and not as intermediates; 2) zero ratings of neither-nor apply to a wide range of images for above-below and in front-behind and this is consistent with the fact that these dimensions, according to BSK taxonomy, have a single intermediate (they belong to the RPR type structure); 3) zero ratings of neither-nor were
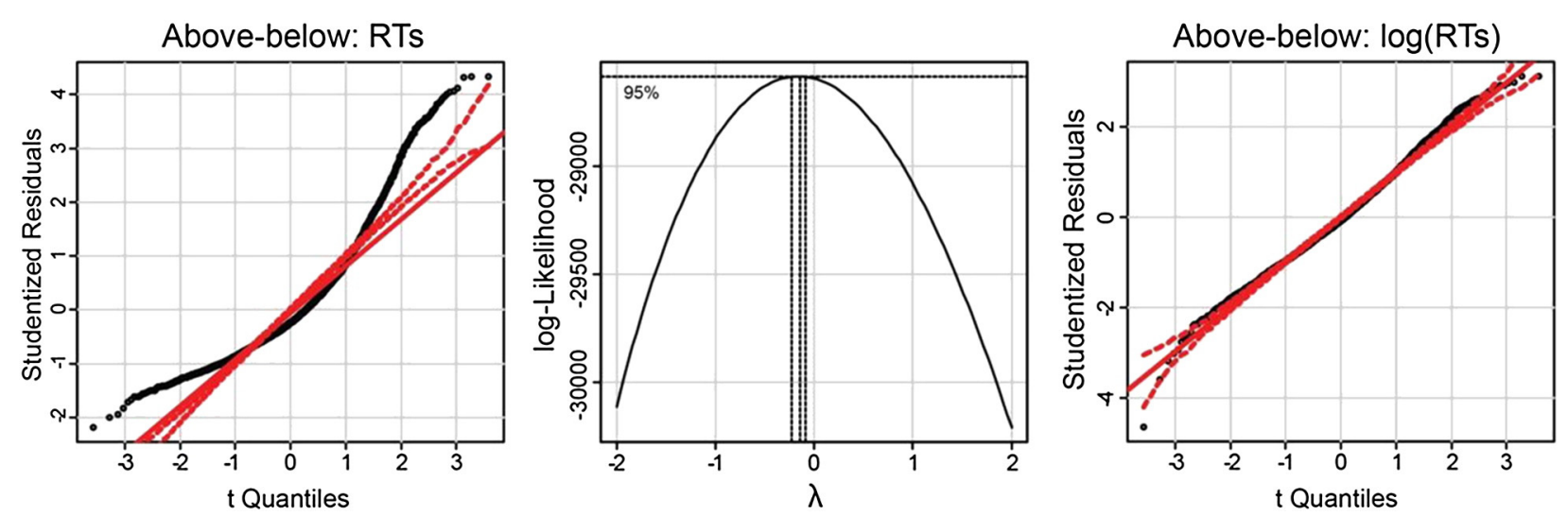

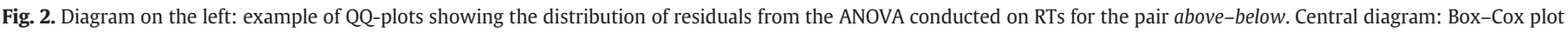

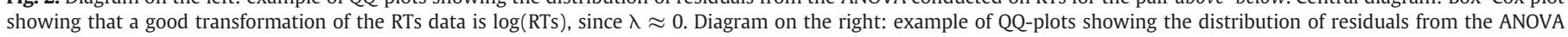
conducted on $\log$ (RTs) for the pair above-below. 

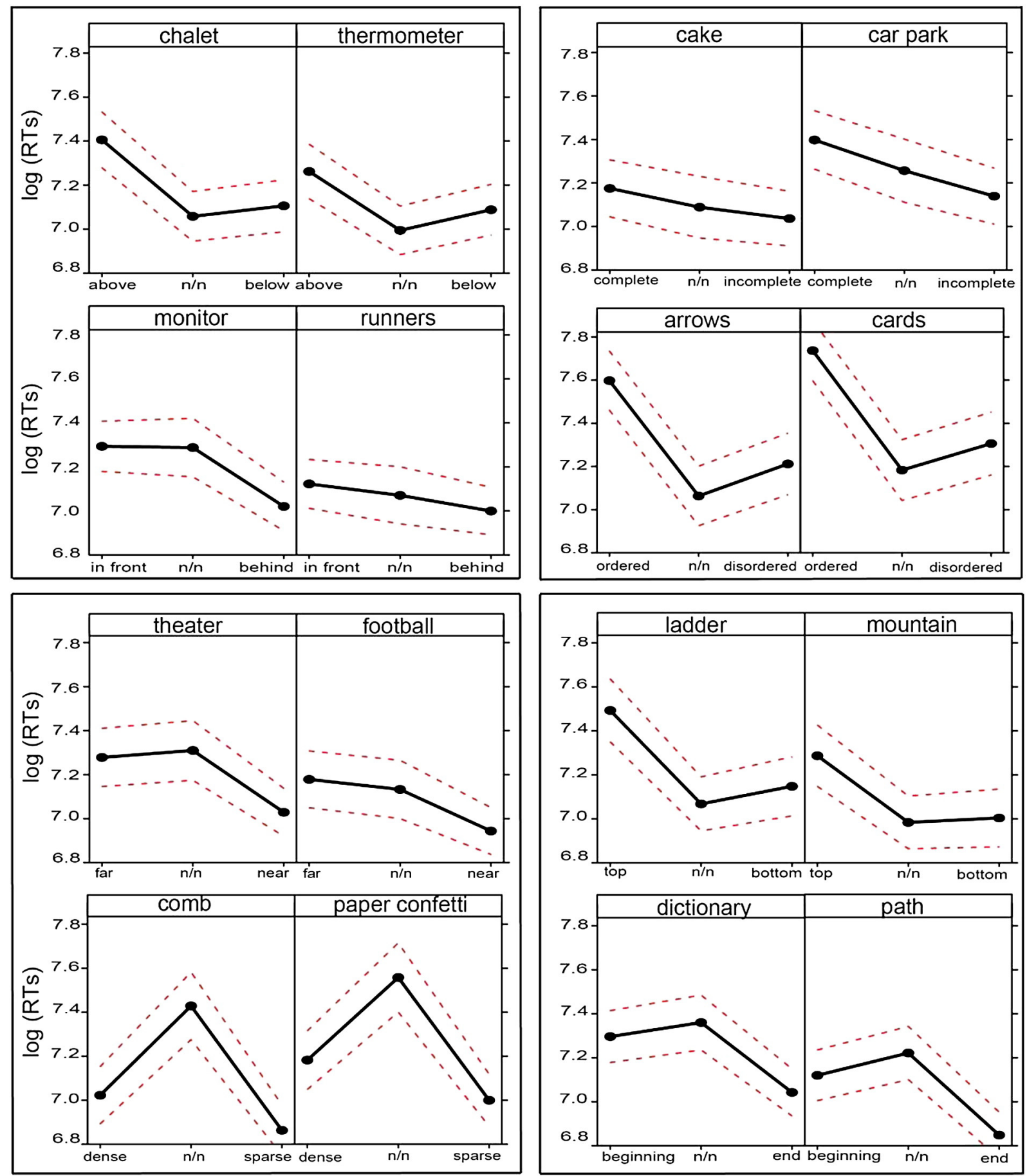

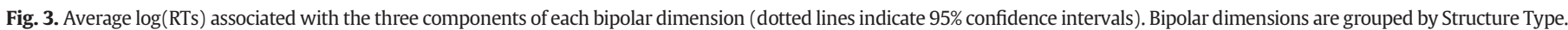

associated with the entire series of images for ordered-disordered for the set of cards as this is in fact a dimension that, according to BSK taxonomy, is characterized by the absence of intermediates (PNR) and zero ratings for intermediates throughout the whole series should have been similarly found for the other object (arrows) and also for the dimension completeincomplete, but this was not the case. This is a discrepancy which needs to be thought about and further investigated (see studies 2 and 3).

With regard to pole properties: 1) in general, zero ratings of a polar property characterized the images which were at the opposite extreme of the series; for example, zero ratings of near applied to images representing the region towards the pole far; this confirms, on a perceptual level, the rule of the mutual exclusion of opposites, i.e. when one property is visible with maximum salience the opposite property is not visible; 2 ) zero ratings of the opposite polar property characterized several images in the series which, according to SBK taxonomy, have a single intermediate point splitting the domain in two halves (i.e. dimension type RPR: above-below and in front-behind). The distribution of zero ratings that was found confirms that for these dimensions a polar property, e.g. above, certainly does not apply either to the images representing the opposite pole or to the central intermediate; similar behavior also characterized end-beginning and top-bottom (PRP according to BSK) where zero ratings cover more than half of the series - even though the extension of zero ratings is not as extended as expected given the characterization of these poles in terms of point properties (this discrepancy also deserves attention - see studies 2 and 3). 

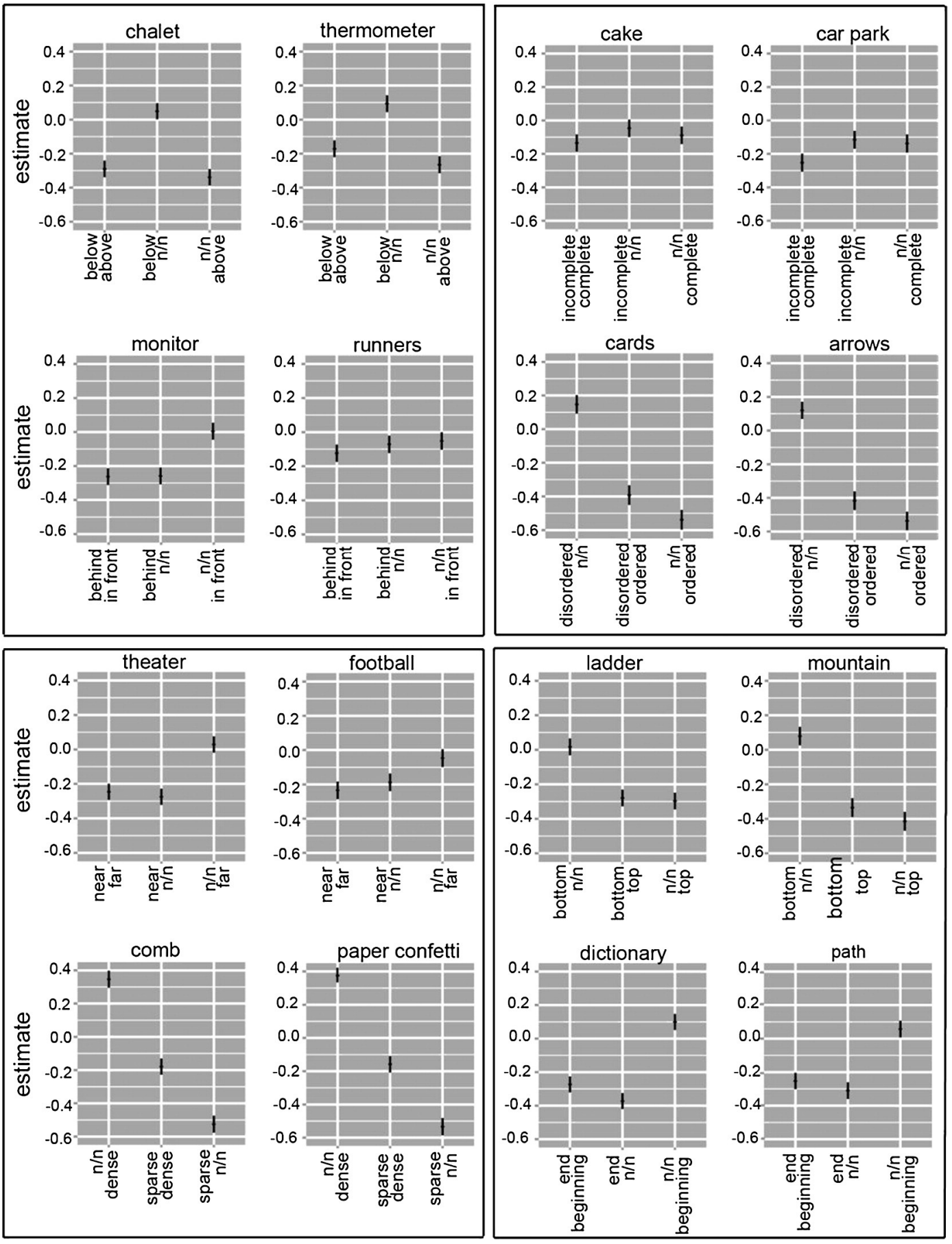

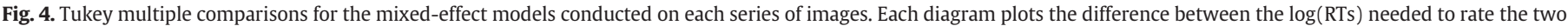

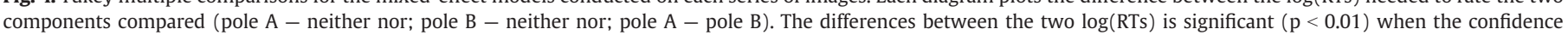
interval does not include 0 ).

When responses fell into two different groups, this usually happened because one group of responses followed a linear trend, i.e. participants judged that the evidence of the property increased constantly throughout the series of images (and this is what one would expect for graded properties). Conversely, the other group of responses followed a distribution that is more typical of binary properties (which are either present or absent).

In summary, for dimensions belonging to the Range-Point-Range structure (top-left quadrant in Fig. 5) the results were compatible with the predictions: there was clear evidence that the central stimulus of the series was perceived as an intermediate. Some responses also indicated the perception of another one or two stimuli as intermediates, but it is clear that the rest of the stimuli were unambiguously perceived as either pole $a$ or pole $b$. This behavior was consistent for all of the 4 pairs analyzed.

For the Range-Range-Range structure (bottom-left quadrant of Fig. 5), the two dimensions analyzed behaved in a way that was generally compatible with the predictions regarding the poles. An extended number of stimuli showed the polar property increasing/ decreasing in a linear manner. The normal distribution of the ratings for neither near-nor far was also in agreement with BSK taxonomy. In contrast, for dense-sparse, the intermediates were not well characterized: they were definitely not perceived at the two extremes of the series, but their distribution around the regression line in the central 
stimuli of the series is extremely widespread, indeed almost random. ${ }^{3}$

The behavior of dimensions belonging to the Point-Range-Point structure (right-bottom quadrant of Fig. 5) reflected in part what we expected. Many of the images in the series were perceived as intermediates. The range was more extended for the dimensions belonging to this typology than for any other dimension considered in the study. What was missing was confirmation of the perception of the two poles as points. In fact only a subgroup of participants perceived the property in a small number of figures and declared it to be absent in all the others; for another subgroup of participants, the ratings of the polar properties decreased in a linear manner.

In dimensions belonging to the Point-No intermediates-Range structure (top-right quadrant of Fig. 5), the behavior of only one component (incomplete, disordered) was found to vary gradually (almost in a linear fashion) as in fact had been expected. The behavior of the other two components was not in agreement with what had been expected based on BSK taxonomy. As in the two previous structures where a Point component was involved (RPR and PRP), the point poles (complete and ordered) turned out to be graded and not singular. Moreover, despite the fact that these dimensions had been expected to have no intermediates, participants did not categorically deny that they perceived intermediacy, but in fact admitted that they perceived its presence. Only in the case of ordered-disordered (applying to the set of arrows) did a few participants give ratings of zero intermediacy to all the figures in the series.

\subsubsection{What happens to the two poles when intermediates are maximally} evident?

In order to further explore the features of intermediates and the differences between them associated with the various types of dimensions, we carried out an additional step in the analyses. We transformed the data from a continuous to a category scale (with 5 categories: 0-4) and focused only on the stimuli for which the most frequent category (the mode) of intermediacy was the maximum value on the new scale (i.e. 4). We wondered what happens to the ratings of the poles in these cases.

This analysis helped us to see further differences between the various types of dimension. It showed in fact that for one type (RPR), maximum evidence of intermediacy excluded the perception to any degree of one or the other pole (see Fig. 6 above-below: when the mode for intermediates is 4 , the mode for both pole $a$ and pole $b$ is 0 ). This was not true for the other types of contrary: for type PRP contraries, the stimuli with the greatest evidence of intermediacy (intermediate mode $=4$ ) at the same time showed to a certain degree evidence of one of the two poles, while the other pole was absent (see Fig. 6 at the top-at the bottom). For the RRR and PNR types, the stimuli with the greatest evidence of intermediacy were at the same time described as showing to a certain degree evidence of both pole $a$ and pole $b$ - to an equal degree in some central stimuli, and to different degrees in other stimuli, more towards the poles (Fig. 6, near-far and complete-incomplete).

These results suggest a sharp distinction between dimensions where the three components (pole $a$, intermediates, pole $b$ ) are disjunctive sets (RPR), and dimensions where the three components

\footnotetext{
${ }^{3}$ Looking for possible explanations for this result, we thought that a first likely critical aspect might be the homogeneity or non-homogeneity of the pattern, i.e. whether the elements forming the textures are regularly distributed (as in a comb) or equidistant (as in paper confetti). If they are not regular, empty spaces prevail in some parts of the pattern while in other areas, where the elements are many and close, they appear dense. This hypothesis would lead to expect neither dense nor sparse to be more ambiguous when applied to non-homogenous patterns (except the extremes) if one considers that the pattern is dense in some parts, and sparse in others. Another critical aspect (possibly independent of homogeneity) may be that in dense-sparse the quantity of the elements co-varies with the distribution of the elements in space. For extreme values (when the elements are very few or when the elements are a lot) how the elements are distributed is likely a component that counts less in determining the appearance of dense-sparse; in the quantification of intermediates, how the elements are distributed might be a critical variable. Of course these are hypotheses to test.
}

overlap (PRP, RRR and PNR). This overlap can be between the set of intermediates and one of the two poles and this is always the case for PRP (for example, something can be perceived to be simultaneously both to a certain degree neither at the top nor at the bottom and to a certain degree at the top). The overlap may also involve the set of intermediates and both poles. This applies to dimensions belonging to the RRR structure (what is perceived as neither near nor far can at the same time be perceived as slightly near and slightly far) and the PNR structure (what is perceived as neither ordered nor disordered can at the same time be perceived as slightly ordered and slightly disordered).

\section{Study 2}

Study 1 demonstrated a) that intermediates are highly evident (in any case more than pole properties) in some of the stimuli presented, b) that in general their recognition and assessment in terms of quantification is not more time consuming than the recognition and assessment of polar properties, c) that there are regularities in the presence versus exclusion to a certain degree of one (or both) polar properties when intermediates are experienced, depending on the type of dimension, and finally d) that responses turned out to be more graded than expected: poles and intermediates which had been expected to be point properties often turned out to have a (more or less extended) gradable structure. Moreover, instead of the absence of intermediates which had been expected for dimension type PNR, gradable ratings of intermediates were found for 3 out of the 4 pairs studied.

Study 2 was designed to test whether the latter two discrepancies (described in point d) might be due to the task used. The same series of images as those presented in study 1 were used in study 2 in a classification task. Participants were asked to decide, for each image, if it showed property $a$, property $b$ or an intermediate between them (neither $a$ nor $b$ ). If the structures of the poles and of the intermediates resulting from this study are consistent with BSK taxonomy, this will prove that the difference between the two tasks is critical.

\subsection{Method}

\subsubsection{Participants}

64 undergraduate students aged 20 to 31 (divided into two groups).

\subsubsection{Materials}

The same series of images as those used in study 1 .

\subsubsection{Procedure}

The experiment was conducted in a room at the university. The instructions were projected onto a screen and were also verbally explained by the person conducting the experiment. Participants were asked to look at the images forming each series and, using a form to record their responses, to indicate whether the property shown was pole $a$, pole $b$ or neither $a$ nor $b$. Each image remained on the screen for $10 \mathrm{~s}$; after $3 \mathrm{~s}$ of blank screen (during which participants responded) the subsequent image appeared. The images forming each series were presented in the same random order as in study 1 .

Each series was introduced by means of a question (reported on the response form) e.g. "Is the flag at the top, at the bottom or neither at the top nor at the bottom of the mountain?". All questions were formulated in the same way. It was explained that we were interested in understanding which variations of a property from one extreme to the opposite extreme are recognized as pole $a$, which as pole $b$ and which as neither $a$ nor $b$. Participants were asked to rely on their perception but to be as precise as possible. They were told to imagine that their responses would be used to teach intelligent machines how to distinguish spatial properties exactly as humans do. 

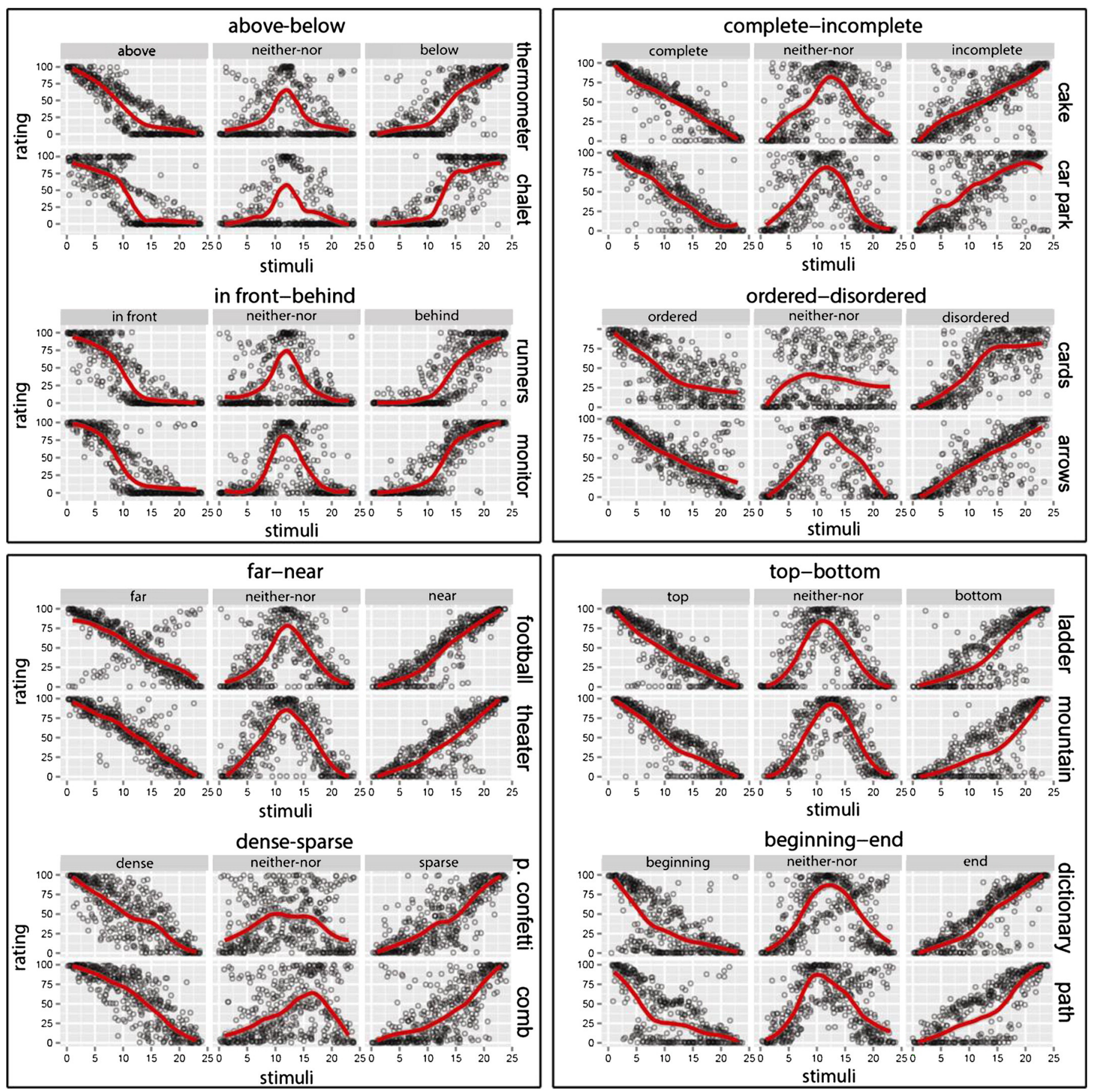

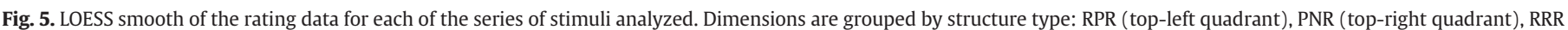
(bottom-left), PRP (bottom-right).

\subsection{Results}

The key-question here is: what range of stimuli did each of the three components elicit? To answer this question we calculated, for each subject and each series of images, the range of stimuli classified, respectively, as pole $a$, intermediates and pole $b$ and then used Linear Mixed Effect Models to study these data. As shown in Fig. 7 (where thick unbroken lines represent the average range for each component while thin unbroken lines represent the most frequent range i.e. the mode), clear differences emerged between types of dimensions, whereas clear similarities emerged within each Type.

Linear Mixed Effect Models - with Type and Component as fixed effects and Dimension and Object as random effects (estimated using ANOVA criterion) - revealed a significant main effect of Component $\left(\mathrm{F}_{(2,3610)}=35.63, \mathrm{p}<0.001\right)$ and a significant interaction Type*component $\left(\mathrm{F}_{(6,7577)}=67.56, \mathrm{p}<0.001\right)$. The interaction is particularly informative. We used Fisher's LSD-Test to focus on the comparisons which were the most interesting for our research questions, i.e. to compare, within each typology, the average range of pole $a$, intermediates and pole $b$. The results of these comparisons are summarized by the expression of equality-inequality reported at the top of each quadrant in Fig. 7 (where $\neq$ indicates that the two components have a significantly different range for $\mathrm{p}<0.05$, and $=$ that they don't). Two typologies (i.e. RPR and PRP) turned out to have a similar extension of the two poles and a significantly different extension of the intermediates (smaller in RPR, bigger in PRP). In the other 
Table 2

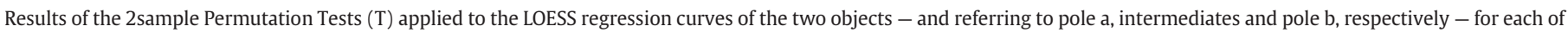
the dimensions analyzed (resampling process $=1000$ ).

\begin{tabular}{|c|c|c|c|}
\hline Dimension & Pole a (obj1 vs 2) & Neither-nor (obj1 vs 2) & Pole b (obj1 vs 2) \\
\hline Above-below & $\mathrm{T}=403, \mathrm{p}=0.9711$ & $\mathrm{~T}=506, \mathrm{p}=0.1805$ & $\mathrm{~T}=441, \mathrm{p}=0.8145$ \\
\hline In front-behind & $\mathrm{T}=385, \mathrm{p}=0.9329$ & $\mathrm{~T}=436, \mathrm{p}=0.9167$ & $\mathrm{~T}=391, \mathrm{p}=0.4256$ \\
\hline Complete-incomplete & $\mathrm{T}=519, \mathrm{p}=0.7669$ & $\mathrm{~T}=594, \mathrm{p}=0.7597$ & $\mathrm{~T}=556, \mathrm{p}=0.3187$ \\
\hline Ordered-disordered & $\mathrm{T}=446, \mathrm{p}=0.3152$ & $\mathrm{~T}=603, \mathrm{p}=0.1259$ & $\mathrm{~T}=570, \mathrm{p}=0.6684$ \\
\hline Far-near & $\mathrm{T}=559, \mathrm{p}=0.9826$ & $\mathrm{~T}=519, \mathrm{p}=0.4052$ & $\mathrm{~T}=459, \mathrm{p}=0.798$ \\
\hline Dense-sparse & $\mathrm{T}=518, \mathrm{p}=0.2909$ & $\mathrm{~T}=577, \mathrm{p}=0.841$ & $\mathrm{~T}=432, \mathrm{p}=0.6424$ \\
\hline Top-bottom & $\mathrm{T}=472, \mathrm{p}=0.7866$ & $\mathrm{~T}=565, \mathrm{p}=0.5631$ & $\mathrm{~T}=360, \mathrm{p}=0.5062$ \\
\hline Beginning-end & $\mathrm{T}=330, \mathrm{p}=0.9544$ & $\mathrm{~T}=624, \mathrm{p}=0.8788$ & $\mathrm{~T}=401, \mathrm{p}=0.6649$ \\
\hline
\end{tabular}

two typologies (RRR, PNR) the poles turned out to be asymmetric with one more extended than the other. Moreover, in type RRR the extension of the intermediate regions is either similar to that of one pole or slightly less extended than both whereas in PNR the range of intermediates is in between that of the two poles when considering the average range but drops to zero stimuli (in agreement with BSK taxonomy) when the most frequent range is considered.

In brief, the dimension structures in study 2 turned out to be in line with BSK classification. For type RPR (above-below and in front-behind) the recognition of intermediacy occurred for only one central stimulus, while all other stimuli were consistently referred to as displaying either one pole or the other. Type PNR (complete-incomplete and ordereddisordered) were described as consisting almost entirely of variations of one pole, since a single image was associated with the opposite pole; with regard to intermediates, the most frequent response (thin line) is in agreement with SBK classification, i.e. absence of intermediates, while the average range (thick line) suggests something different. For type PRP (at the top-at the bottom and beginning-end) the two extreme properties showed, respectively, one property and its opposite, while all other stimuli were perceived as variations of intermediates. With type RRR (near-far and dense-sparse), all three components applied to a range of stimuli as in BSK but in our study the "smaller" pole, i.e. sparse and near, was more extended than the opposite pole, dense and far, while in BSK it was the other way round. This inversion might be due to the kind of stimuli presented: images were limited by the size of the computer screen - they could not therefore be quantitatively as extensive as they would be in ecological settings such as those used for the BSK taxonomy.

\section{Study 3}

Studies 1 and 2 left us with a thought-provoking question concerning the difference between the two studies in terms of gradability: properties defined as point properties in study 2 were graded in study 1 , and intermediates described as non-existent in study 2 were described as present and graded in study 1 . Before coming to a conclusion regarding this difference, we decided to carry out a further rating task, as in study 1, but using a simplified version. Given that our main interest here concerned the structure of intermediates, we limited quantification to the intermediate component, asking participants to look at the images forming each series and to respond to the question: "to what extent is the property [neither a nor $b$ ] visible"? If the property was evident to some extent, participants were then requested to quantify this. If their answer was zero, one of the two poles was therefore visible, and they were requested to indicate which. Since participants were not being asked to rate the same images for the three components (as in study 1), the possibility that polar ratings might interfere with the intermediate ratings can be
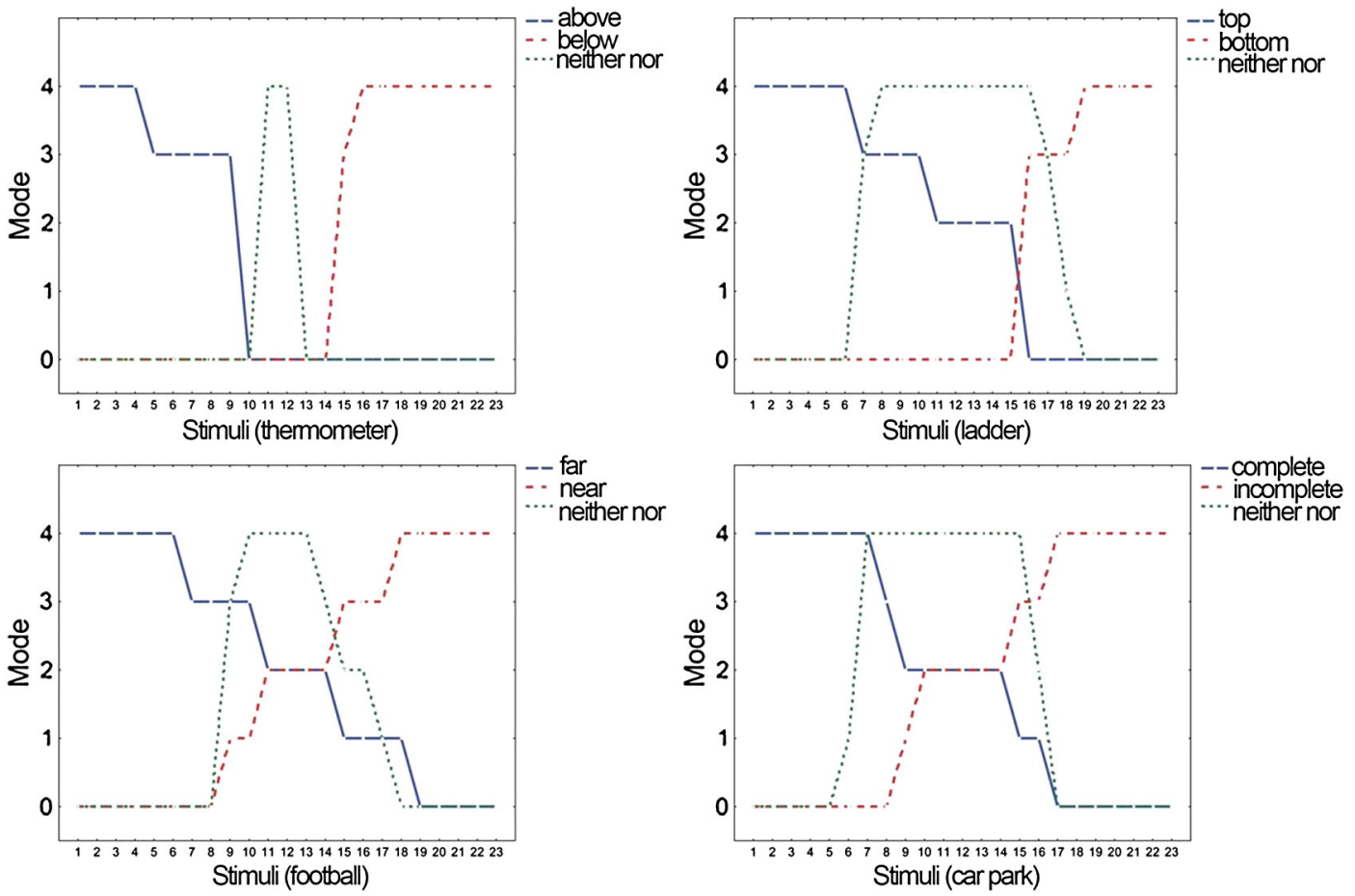

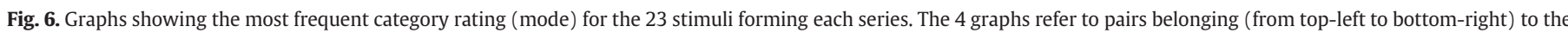
RPR, PRP, RRR and PNR structures respectively. 
excluded. If, despite this, the results from study 3 confirm what was found in study 1 , this will reinforce the conclusion that quantification and classification lead to different structures (with interesting theoretical and methodological implications).

\subsection{Method}

\subsubsection{Participants}

88 undergraduate students (aged 19 to 31 ), divided into two groups, 47 in group 1, 41 in group 2.

\subsubsection{Materials}

The same pairs of opposites and series of images as those used in studies 1 and 2 . The series (and the 23 images within each series) were presented in the same random order as in the previous studies.

\subsubsection{Procedure}

The experiment was conducted in a room at the University. The two groups participated in the study separately at the beginning of a class which was unrelated to the topic of the research. Participants were asked to look at the images forming each series (each image was projected on a screen for $10 \mathrm{~s}$ ) and to rate to what extent the property neither $a$ nor $b$ was visible (e.g. "to what extent is the property neither at the top nor at the bottom visible?"). A 0-10 rating scale was used.

A response sheet with 8 tables (one for each series) was provided. There was a list of numbers corresponding to the 23 images and 3 columns. The central column was for ratings of intermediacy and if the rating was 0 , participants were asked to mark a cross in the column to the left or to the right of the central column corresponding respectively to pole $a$ or pole $b$.

The questions, which changed at the beginning of each series, were projected and also read aloud by the experimenter before the presentation of the images. Participants were asked to focus on what they perceived.

\subsection{Results}

Fig. 8 shows a comparison between the ratings of intermediates in studies 1 and 3 (data from Study 1 had previously been recoded on a scale $0-10$, in order to use the same scale as study 3 ). As in study 1 , LOESS regression lines were drawn on the ratings.

The trend of responses turned out to be very similar in the two studies, as Fig. 8 shows and as the results of the 2-sample Permutation tests used to study the difference between the points forming the LOESS curves in studies 1 and 3 confirmed (see Table 3 ). It should be remembered that the 23 stimuli forming each series were presented in random order so both the consistency of the responses within each series (smoothness of the curves) and the similarity between the two studies are indices of the robustness of the responses.

In general, data from study 3 confirmed that in a rating task properties (intermediates in this case) which in a classification task would be described as ungradable are described as being gradable. In the same way, properties (intermediates) that in a classification task would be described as non-existent may be described to be present to a certain degree. The two different distributions of responses previously found for the intermediate component in RPR type structures (above-below and in front-behind) were confirmed as was the almost random distribution of responses for neither dense nor sparse.

\section{Final discussion}

In this article we looked for experimental evidence supporting the hypothesis that intermediates deserve to be considered as perceptual components of the structure of a dimension as much as the poles are. This hypothesis is based, in general, on the idea that that opposites are cognitive structures grounded in how properties appear in terms of qualitative invariance or significant variations (Bianchi \& Savardi, 2008a, 2008b, 2012; Savardi \& Bianchi, 2009; Savardi, Bianchi, \& Bertamini, 2010). It develops a tenet - that of the foundation of cognition in perception and action - already discussed in previous literature with regard to other cognitive structures and functions (for a review see Pecher \& Zwaan, 2005).

Aristotle (Methaphysics, 1057a18-1057b04; Physics, 227a07-10; 229b15-20) already had an intuition that the species of opposition which was most useful to refer to the empirical world was contrariety since, according to his definitions, contrariety admitted intermediates whereas other species of opposition did not. This article in a sense confirms his intuition.

We investigated by means of experimentation the direct experience of properties specifically recognized by observers as neither variations of a pole nor variations of the opposite pole by focusing on spatial experiences. Three main results emerged from these experiments. Firstly, the response times in study 1 suggest that, for adults, intermediates are no more difficult to recognize and rate than polar properties when pictures showing various ecological scenes are observed. This finding is relevant since it allows us to exclude the possibility that the recognition of intermediates is not as direct as that of the two opposites (i.e. it is not derived by means of a mental process of double exclusion of pole $a$ and of pole $b$ as the linguistic structure neither $a-$ nor $b$ might lead one to think).

Secondly, the findings proved that participants were generally in agreement when rating the extent to which intermediates were visible. This consistency emerged within each series of images: despite the fact that the 23 images forming each series were presented in random order, the shape of the functions describing the average rating of intermediacy was usually very smooth. Furthermore, there was a similarity in the ratings given to the same series of images by two different samples of participants (i.e. study 1 versus study 3 ).

A third, more controversial, result concerns the number of images (within each series) showing greater evidence of neither pole a nor pole $b$ rather than pole $a$ or pole $b$. It ranged from one single picture to most of the 23 pictures forming each series, depending on the type of the dimension (RPR, PRP, RRR, PNR). However, even though the responses in study 2, where a classification task was used, fitted in almost perfectly with the taxonomy suggested in previous literature based on a different type of task (Bianchi, Savardi, \& Kubovy, 2011) and indeed fitted perfectly when the most frequent response (mode) was considered, two systematic differences emerged when a rating task was used (i.e. in studies 1 and 3 ). The first systematic difference consisted of a generalization of the structure of a range to properties classified as points; this was not specific to intermediates but was true also for the poles. The second systematic difference concerned ratings other than zero applying to intermediates which in classification tasks had been described as non-existent (i.e. to dimensions type PNR).

\subsection{Potential impact of the results}

The difference between the two tasks is interesting for both theoretical and methodological reasons. It tells us that people who are asked to give quantitative ratings tend to graduate what in other conditions they would describe and deal with as a binary variable (i.e. either present or absent). Our findings, in a sense, support Paradis's (2008) idea - which challenges widespread assumptions in linguistics and cognitive linguistics - that degree is not a grammatical phenomenon which is a characteristic only of certain types of word classes, but rather can be associated with most meanings. In her view, this happens because expressions that at first sight display non gradable content (and therefore do not appear to lend themselves to grading) may have a background structure suggesting gradability that comes in the foreground when linguistic degree 

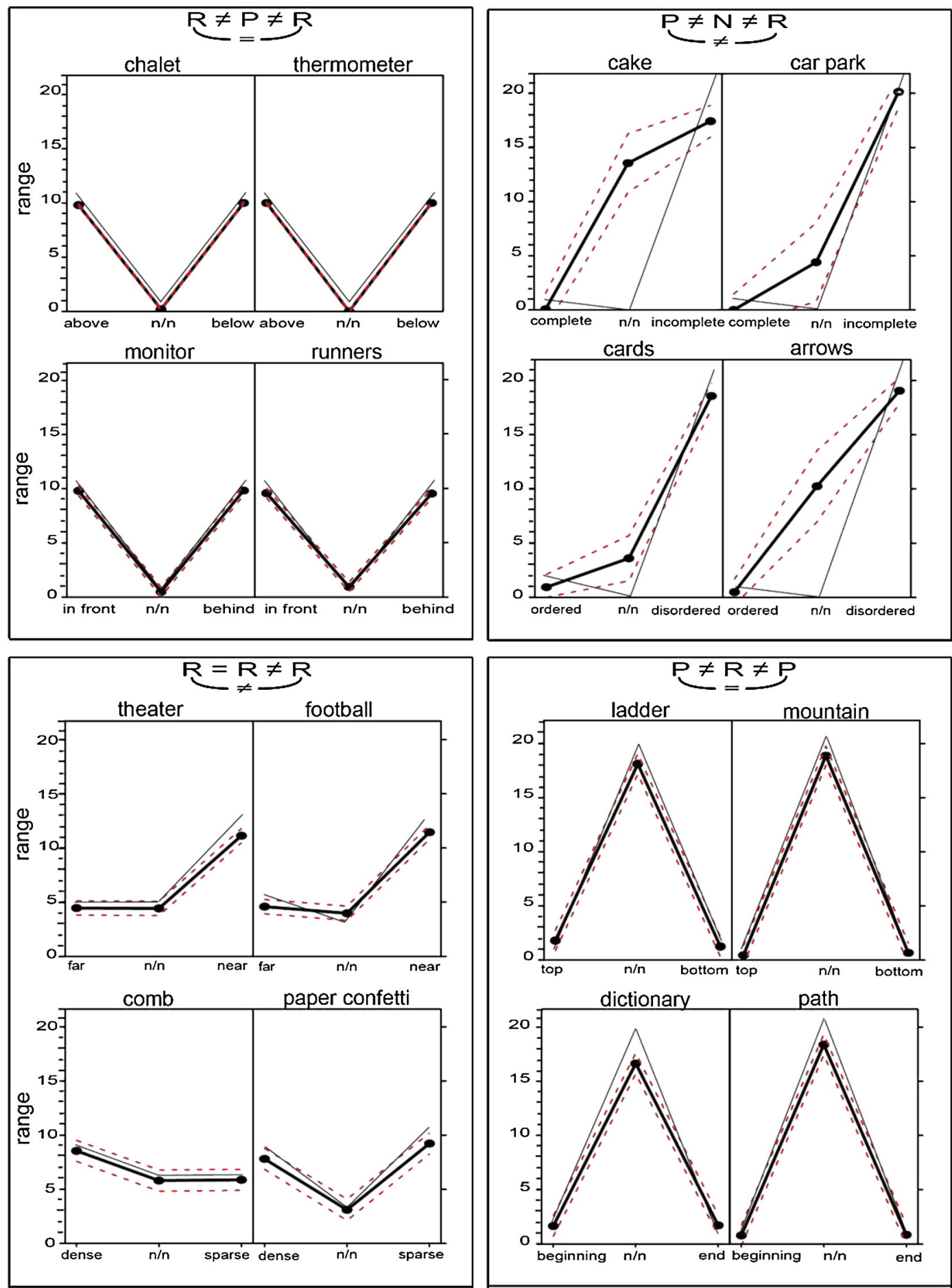

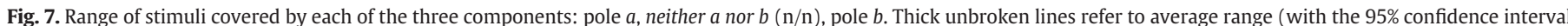

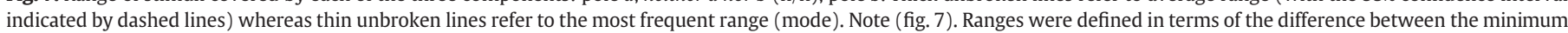

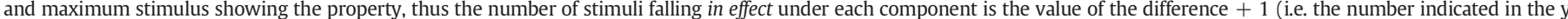

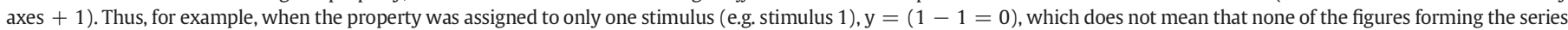
was classified in this way, but that one was.

modifiers are used. An interesting point to be discussed, in our view, is whether only semantics is involved in this process or whether this shift is grounded in perception. Paradis too admits that "what is characteristic of the path of development of degree meanings is the conceptualization of the message as increasingly strongly grounded in the situation" (2008, p. 337). Informal interviews carried out with our participants at the end of studies 1 and 3 proved that participants who responded to the question "to what extent, looking at the cake/ car park, do you perceive the property neither complete nor incomplete?" with a rating of 0 , gave their reason for this saying that, except for the pictures showing a whole cake and a full car park (i.e. complete), all other conditions showed a cake/car park which was incomplete or more or less incomplete. Conversely, those who described the car park as neither complete nor incomplete acknowledged that they had shifted from rating complete-incomplete to full-empty when referring to the car park and to the amount of cake remaining ( $a$ lot-a little) when 
(study 1)

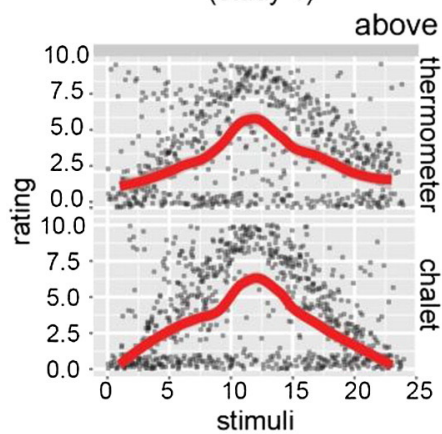

in front - behind
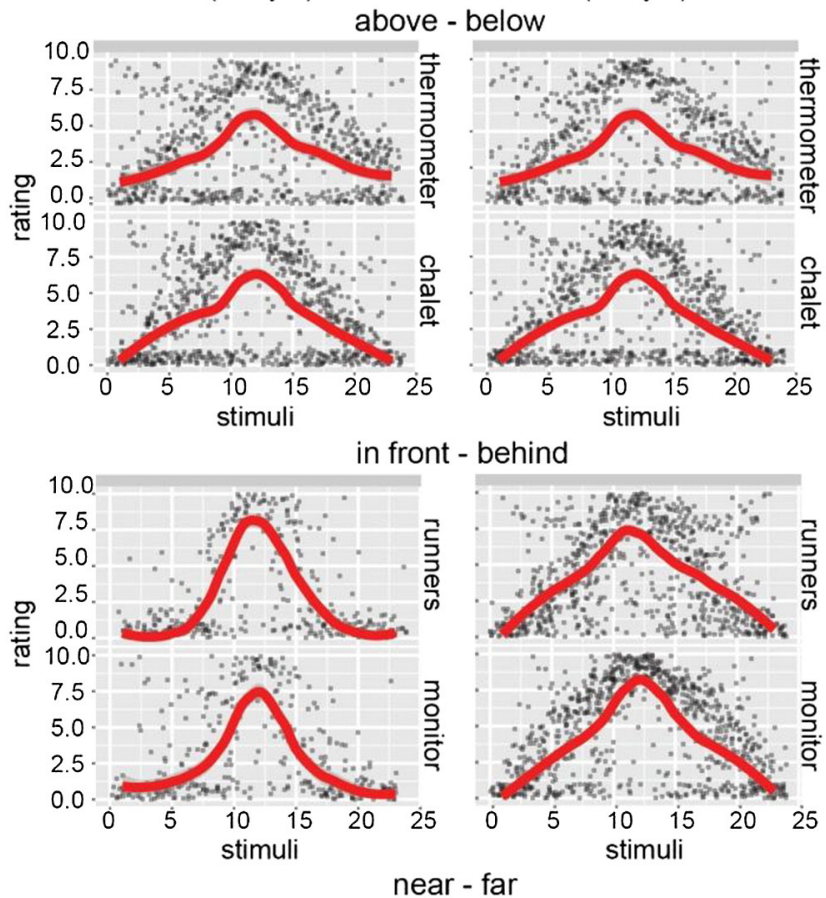

(study 3)
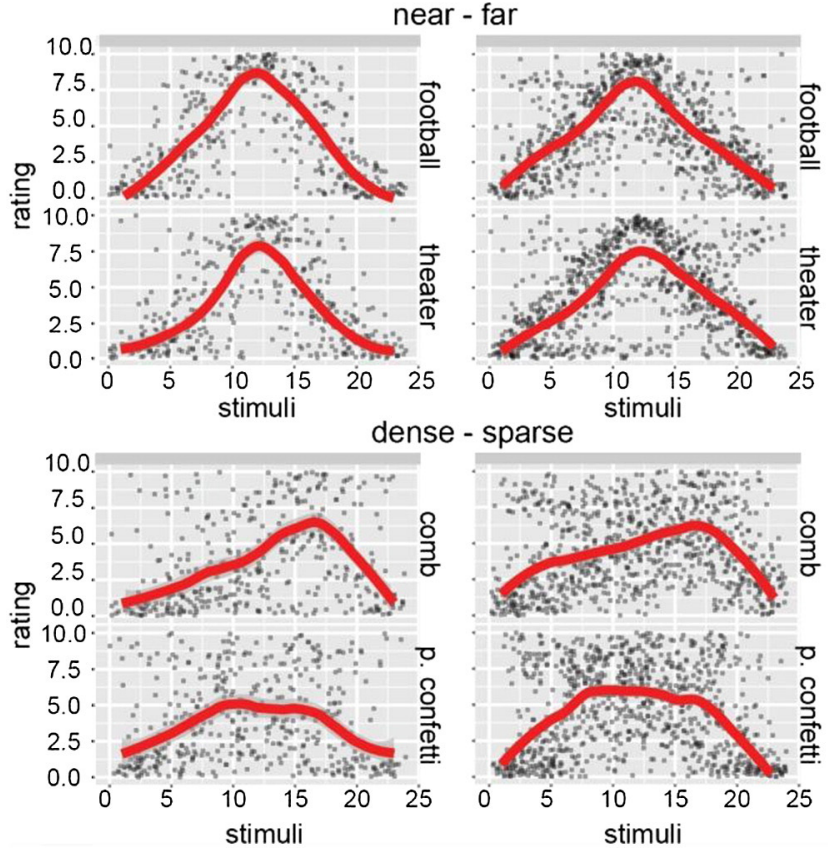
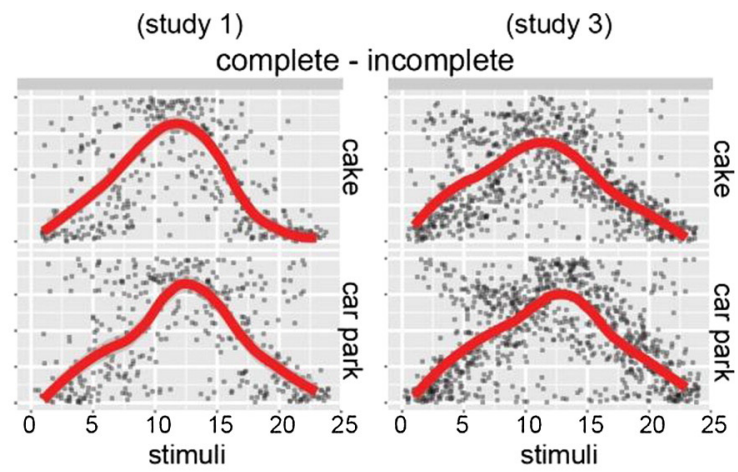

ordered - disordered

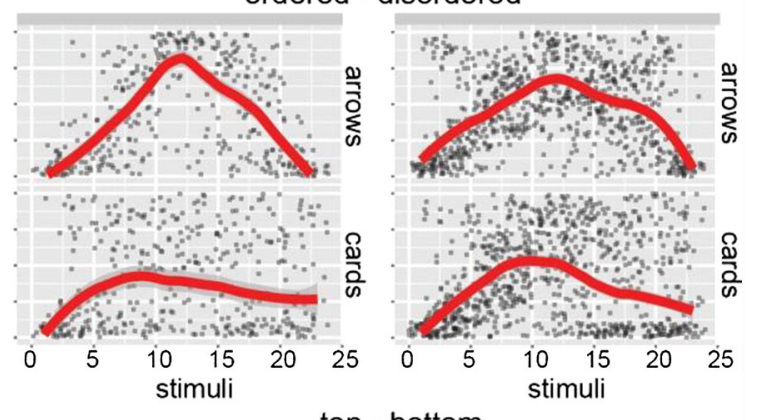

top - bottom
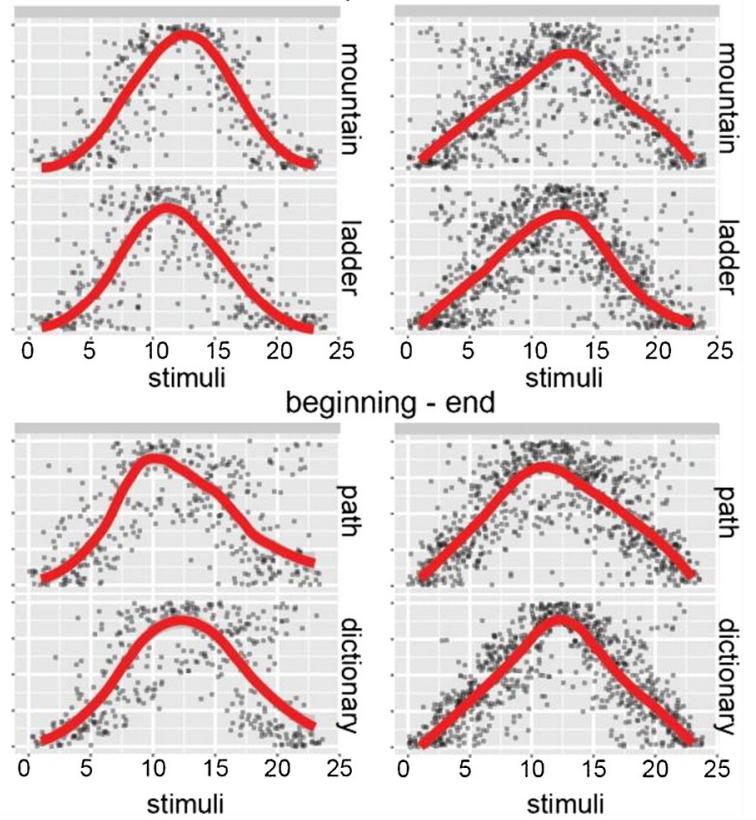

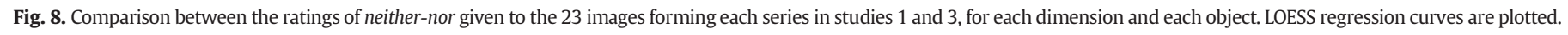

referring to the cake. And in fact, neither full nor empty and neither a lot nor a little apply to many images of the series (full-empty has a PRP structure; a lot-a little has a RRR structure). A similar shift occurred for point properties concerning the poles. For example, participants who rated at the top as visible to a certain degree when looking at a flag which was beyond the middle of a mountain but not yet at the top of it (and often at a considerable distance from it) admitted that they had shifted from the dimension at the top-at the bottom to the dimension high-low (which indeed has a RRR structure).

We suggest that the multidimensionality of phenomenal experiences is the key aspect here. It has been already shown that, when applied to ecological scenes, perceptual judgments of large-small, high-low, longshort, wide-narrow are not unidimensional (Bianchi, Savardi, \& Burro, 2011). The shifts emerging in studies 1 and 3 manifest an interlace between dimensions which comes to the fore when properties are 'experienced' and not simply 'defined' in terms of a concept or meaning: it is not by chance that the shift away from complete-incomplete ends up in full-empty when the event under observation has the visual structure of a car park (where discrete elements populate a delimitated space and every element has its own place) but not when the object has a continuous visual structure (the cake). The consideration that this interlace between dimensions is embedded in the perceptual structure of what is being observed and thus is objective not subjective, might reinforce some intuitions expressed in cognitive linguistic literature (e.g. Paradis, 2008; Traugott \& Dasher, 2005).

The second potential impact of our results concerns the models and taxonomies of antonymy and opposites produced in cognitive semantics and inspired by the genuine desire to link linguistic 


\section{Table 3}

Results of the 2-sample Permutation Tests applied to the LOESS regression curves of ratings of neither-nor in studies 1 vs 3 , for each of the dimensions analyzed and each object (resampling process $=1000$ ).

\begin{tabular}{lll}
\hline Dimension & $\begin{array}{l}\text { Ratings of intermediates } \\
\text { Object 1 (study 1 vs 3) }\end{array}$ & $\begin{array}{l}\text { Ratings of intermediates } \\
\text { Object 2 (study 1 vs 3) }\end{array}$ \\
\hline Above-below & $\mathrm{T}=596, \mathrm{p}=0.993$ & $\mathrm{~T}=680, \mathrm{p}=0.987$ \\
In front-behind & $\mathrm{T}=456, \mathrm{p}=0.112$ & $\mathrm{~T}=397, \mathrm{p}=0.052$ \\
Complete-incomplete & $\mathrm{T}=572, \mathrm{p}=0.991$ & $\mathrm{~T}=580, \mathrm{p}=0.875$ \\
Ordered-disordered & $\mathrm{T}=606, \mathrm{p}=0.563$ & $\mathrm{~T}=616, \mathrm{p}=0.406$ \\
Far-near & $\mathrm{T}=586, \mathrm{p}=0.740$ & $\mathrm{~T}=544, \mathrm{p}=0.359$ \\
Dense-sparse & $\mathrm{T}=544, \mathrm{p}=0.142$ & $\mathrm{~T}=632, \mathrm{p}=0.296$ \\
Top-bottom & $\mathrm{T}=564, \mathrm{p}=0.893$ & $\mathrm{~T}=569, \mathrm{p}=0.7414$ \\
Beginning-end & $\mathrm{T}=626, \mathrm{p}=0.8236$ & $\mathrm{~T}=610, \mathrm{p}=0.8035$ \\
\hline
\end{tabular}

structures to their most likely cognitive counterparts (e.g. Cruse \& Togia, 1995; Paradis \& Willners, 2011). Our results demonstrate the need to think carefully about the region which lays in between poles which cannot be modeled simply as weaker gradations of one or the other pole: some dimensions have a range of instances (others only one point experience) that are a third component - neither pole $a$, nor pole $b$ - situated between the two poles.

Finally, it seems to us that the presence or absence of an intermediate region and its extension might also have implications for studies from the perspective of an experiential view of language comprehension which aims to predict the representation activated by a negative sentence (Kaup, Lüdtke, \& Zwaan, 2006; Kaup, Yaxley, Madden, Zwaan, \& Lüdtke, 2007; Kaup, Zwaan, \& Lüdtke, 2007). There has been growing evidence in literature that understanding a text activates the construction of mental simulations which are considered to be experiential in nature (Pecher \& Zwaan, 2005; Pulvermüller, 2002; Richardson, Spivey, Barsalou, \& McRae, 2003). In this framework, it has been suggested that comprehending a negative sentence (e.g. "the umbrella is not open") implies a two-step process of representation (Hasson \& Glucksberg, 2006; Kaup et al., 2006; Mayo, Schul, \& Burnstein, 2004). Kaup et al. $(2006,2007)$ found that $250 \mathrm{~ms}$ after reading a text containing a negation, a facilitation effect occurred when participants were shown a picture of this negated state of affairs (in the example a closed umbrella), but if the picture was shown after $1500 \mathrm{~ms}$, there were shorter response times for the picture representing the positive actual state of affairs (an open umbrella). The authors regard these results to be not only proof that processing negated sentence predicates involves a two-phase process (corresponding to the activation of two opposite representations, the negated state of affairs followed by the positive state of affairs), but also as proof that $1500 \mathrm{~ms}$ after reading a sentence such as "the door was not open", people mentally recreate a closed door. Now, open-close are contraries without intermediates (they belong to the PNR structure). What if the negated sentence relates to dimensions with a wide range of intermediates, such as at the top-at the bottom? Given the sentence "the flag is not at the top of the mountain", are we sure that the facilitation effect, after $1500 \mathrm{~ms}$, would occur with an image of a flag at the bottom of the mountain or with an image of a flag in an intermediate position? Would the same happen with dimensions characterized by a single intermediate, such as in front-behind? The phenomenological structure of opposites plays a role in the referential understanding of negation (Bianchi, Savardi, Burro, \& Torquati, 2011; Fraenkel \& Schul, 2008; Paradis \& Willners, 2006). It might be worth investigating whether it affects various other forms of representation processing as well.

\section{Acknowledgments}

We would like to thank Michael Kubovy and another anonymous reviewer for the comments they made on a previous version of this manuscript which significantly contributed to its improvement.

\section{References}

Aristotle (ed. 1984). Metaphysics. In: J. Barnes (ed.), The Complete Works of Aristotle: The Revised Oxford Translation. Princeton, NJ, USA: Princeton University Press.

Aristotle (ed. 1984). Physics. In: J. Barnes (ed.), The Complete Works of Aristotle: The Revised Oxford Translation. Princeton, NJ, USA: Princeton University Press.

Arnheim, R. (1988). The power of the center: A study of composition in the visual arts. Berkeley, CA: University of California Press.

Bianchi, I., \& Savardi, U. (2006). Oppositeness in visually perceived forms. Gestalt Theory, 4, 354-374.

Bianchi, I., \& Savardi, U. (2008a). The perception of contraries. Roma, IT: Aracne.

Bianchi, I., \& Savardi, U. (2008b). The relationship perceived between the real body and the mirror image. Perception, 5, 666-687.

Bianchi, I., \& Savardi, U. (2012). The cognitive dimensions of contrariety. In J. -Y Bezieau, \& G. Payette (Eds.), The Square of Opposition. A general framework for cognition (pp. 443-470). New York,NY: Peter Lang Publishing Group.

Bianchi, I., Savardi, U., \& Burro, R. (2011). Perceptual ratings of opposite spatial properties: Do they lie on the same dimension? Acta Psychologica, 138(3), 405-418.

Bianchi, I., Savardi, U., Burro, R., \& Torquati, S. (2011). Negation and psychological dimensions. Journal of Cognitive Psychology, 23(3), 275-301.

Bianchi, I., Savardi, U., \& Kubovy, M. (2011). Dimensions and their poles: A metric and topological theory of opposites. Language \& Cognitive Processes, 26(8), 1232-1265.

Bowers, D., \& Heilman, K. M. (1980). Pseudoneglect: Effects of hemispace on a tactile bisection task. Neuropsychologia, 18, 491-498.

Bracco, F., Bianchi, I., Chiorri, C., Burro, R., \& Savardi, U. (2009). Investigating contraries by means of change detection. In U. Savardi (Ed.), The perception and cognition of contraries (pp. 93-112). Milano, IT: Mc-Graw Hill.

Brooks, J. L., Della Sala, S., \& Logie, R. H. (2011). Tactile rod bisection in the absence of visuo-spatial processing in children, mid-age and older adults. Neuropsychologia, 49(2011), 3392-3398.

Casasola, M. (2008). The development of infants' spatial categories. Current Directions in Psychological Science, 7(1), 21-25.

Casasola, M., Cohen, L. B., \& Chiarello, E. (2003). Six-month-old infants' categorization of containment spatial relations. Child Development, 74, 1-15.

Cattaneo, Z., Fantino, M., Tinti, C., Pascual-Leone, A., Silvanto, J., \& Vecchi, T. (2011). Spatial biases in peripersonal space in sighted and blind individuals revealed by haptic line bisection paradigm. Journal of Experimental Psychology. Human Perception and Performance, 37(4), 1110-1121.

Cavézian, C., Valadao, D., Hurwitz, M., Saoud, M., \& Danckert, J. (2012). Finding centre: Ocular and fMRI investigations of bisection and landmark task performance. Brain Research, 1437, 89-103.

Clancey, W. J. (1997). Situated cognition: On human knowledge and computer representation. New York: Cambridge University Press.

Croft, W., \& Cruse, D. A. (2004). Cognitive linguistics. Cambridge: Cambridge University Press.

Cruse, D. A. (1986). Lexical Semantics. Cambridge: Cambridge University Press.

Cruse, D. A., \& Togia, P. (1995). Towards a cognitive model of antonymy. Lexicology, $1(1), 113-141$.

Dufour, A., Touzalin, P., \& Candas, V. (2007). Rightward shift of the auditory subjective straight ahead in right- and left-handed subjects. Neuropsychologia, 45(2), 447-453.

Fink, G. R., Marshall, J. C., Weiss, P. H., \& Zilles, K. (2001). The neural basis of vertical and horizontal line bisection judgments: An fMRI study of normal volunteers. Neurolmage $14,59-67$.

Fraenkel, T., \& Schul, Y. (2008). The meaning of negated adjectives. Intercultural Pragmatics, $5,517-540$.

Gallace, A., Auvray, M., \& Spence, C. (2007). The modulation of haptic line bisection using a visual illusion and optokinetic stimulation. Perception, 36, 1003-1018.

Giora, R., Heruti, V., Metuki, M., \& Ofer, F. (2009). When we say no we mean no: On negation in vision and language. Journal of Pragmatics, 41(11), 2159-2184.

Hasson, U., \& Glucksberg, S. (2006). Does understanding negation entail affirmation? An examination of negated metaphors. Journal of Pragmatics, 38, 1015-1032.

Jewell, G., \& McCourt, M. E. (2000). Pseudoneglect: A review and meta-analysis of performance factors in line bisection tasks. Neuropsychologia, 38, 93-110.

Jones, S. (2002). Antonymy. A corpus based perspective. London: Routledge.

Kaup, B., Lüdtke, J., \& Zwaan, R. A. (2006). Processing negated sentences with contradictory predicates: Is a door that is not open mentally closed? Journal of Pragmatics, 38, 1033-1050.

Kaup, B., Yaxley, R. H., Madden, C. J., Zwaan, R. A., \& Lüdtke, J. (2007). Experiential simulations of negated text information. Quarterly Journal of Experimental Psychology, 60, 976-990.

Kaup, B., Zwaan, R. A., \& Lüdtke, J. (2007). The experiential view of language comprehension. How is negation represented? In F. Schmalhofer, \& C. A. Perfetti (Eds.), Higher language processes in the brain: Inference and comprehension processes (pp. 255-288). Mahwah, NJ: Erlbaum.

Kennedy, C., \& McNally, L. (2005). Scale structure and the semantic typology of gradable predicates. Language, 81(2), 345-381.

Kubovy, M. (2003). Phenomenology, psychological. In L. Nadel (Ed.), Encyclopedia of cognitive science (pp. 579-586). Houndmills, Basingstoke, Hampshire, UK: Nature Publishing Group.

Kubovy, M., \& Gepshtein, S. (2003). Grouping in space and in space-time: An exercise in phenomenological psychophysics. In R. Kimchi, M. Behrmann, \& C. R. Olson (Eds.), Perceptual organization in vision: Behavioral and neural perspectives (pp. 45-86). Mahwah, NJ, USA: Erlbaum.

Lee, B. H., Jeong, Y., Kand, S. J., Baek, M. J., Chin, J., Adair, J. C., et al. (2004). Visual search pattern during the line quadrisection task in normal subjects. Experimental Brain Research, 156, 487-494. 
Linkenauger, S. A., Witt, J. K., \& Proffitt, D. R. (2011). Taking a hands-on approach: Apparent grasping ability scales the perception of object size. Journal of Experimental Psychology. Human Perception and Performance, 37(5), 1432-1441.

Lyons, J. (1977). Semantics. Cambridge, UK: Cambridge University Press.

Mannella, F., \& Baldassarre, G. (2007). A neural-network reinforcement-learning model of domestic chicks that learn to localize the centre of closed arenas. Biological Sciances, 362, 383-401.

Masin, S. C. (2008). Voluntary visual attention and phenomenal line length. Perceptual and Motor Skills, 107, 273-287.

Mattingley, J. B., Bradshaw, J. L., Nettleton, N. C., \& Bradshaw, J. A. (1994). Can task specific perceptual bias be distinguished from unilateral neglect? Neuropsychologia, 32(7), 805-817.

Mayo, R., Schul, Y., \& Burnstein, E. (2004). “I am not guilty” vs “I am innocent”: Successful negation may depend on the schema used for its encoding. Journal of Experimental Social Psychology, 40, 433-449.

McCourt, M. E., \& Olafson, C. (1997). Cognitive and perceptual influences on visua line bisection: Psychophysical and chronometric analyses of pseudoneglect. Neuropsychologia, 35, 369-380.

Millar, S., \& Al-Attar, Z. (2000). Vertical and bisection bias in active touch. Perception, $29,481-500$.

Murphy, M. L. (2003). Semantic relations and the lexicon: Antonyms, synonyms and other semantic paradigms. Cambridge, UK: Cambridge University Press.

Ocklenburg, S., Hirnstein, M., Hausmann, M., \& Lewald, J. (2010). Auditory space perception in left- and right-handers. Brain and Cognition, 72(2), 210-217.

Paradis, C. (2000). It's well weird. Degree modifiers of adjectives revised: the nineties. In John Kirk (Ed.), ), Corpora galore: analyses and techniques in describing English (147-160). Amsterdam \& Atlanta: Rodopi.

Paradis, C. (2008). Configurations, construals and change: expressions of degree. English Language and Linguistics, 12(2), 317-343.

Paradis, C., \& Willners, C. (2006). Antonymy and negation-The boundedness hypothesis. Journal of Pragmatics, 38, 1051-1080.

Paradis, C., \& Willners, C. (2011). Antonyms: From conventionalization to meaningmaking. Review of Cognitive Linguistics, 9(2), 367-391.

Pecher, D., \& Zwaan, R. A. (Eds.). (2005). Grounding cognition: The role of perception and action in memory, language, and thinking. Cambridge, UK: Cambridge University Press.
Post, R. B., O'Malley, M. D., Yeh, T. L., \& Bethel, J. (2006). On the origin of vertical line bisection errors. Spatial Vision, 19, 505-527.

Pulvermüller, F. A. (2002). Brain perspective on language mechanisms: From discrete neuronal ensembles to serial order. Progress in Neurobiology, 67, 85-111.

Quinn, P. C. (2005). Developmental constraints on the representation of spatial relation information: Evidence from preverbal infants. In L. A. Carlson, \& E. van der Zee (Eds.), Functional features in language and space: Insights from perception, categorization, and development. New York, NY: Oxford University Press.

Richardson, D. C., Spivey, M. J., Barsalou, L. W., \& McRae, M. (2003). Spatial representations activated during real-time comprehension of verbs. Cognitive Science, 27, 767-780.

Riener, C. R., Stefanucci, J. K., Proffitt, D. R., \& Clore, G. (2011). An effect of mood on the perception of geographical slant. Cognition E' emotion, 25(1), 174-182.

Savardi, U. (Ed.). (2009). The perception and cognition of contraries. Milano, IT: Mc-Graw Hill.

Savardi, U., \& Bianchi, I. (2009). The spatial path to contrariety. In U. Savardi (Ed.), The perception and cognition of contraries (pp. 63-92). Milano, IT: Mc-Graw Hill.

Savardi, U., Bianchi, I., \& Bertamini, M. (2010). Naive prediction of orientation and motion in mirrors. From what we see to what we expect reflections to do. Acta Psychologica, 134(1), 1-15.

Sosa, Y., Clarke, A. M., \& McCourt, M. E. (2011). Hemifield asymmetry in the potency of exogenous auditory and visual. Vision Research, 51(11), 1207-1215.

Tommasi, L., \& Thinus-Blanc, C. (2004). Generalization in place learning and geometry knowledge in rats. Learning \& Memory, 11, 153-161.

Tommasi, L., \& Vallortigara, G. (2000). Searching for the center: Spatial cognition in the domestic chick (Gallus gallus). Journal of Experimental Psychology: Animal Behaviour, 26, 477-486.

Tommasi, L., Vallortigara, G., \& Zanforlin, M. (1997). Young chickens learn to localize the centre of a spatial environment. Journal of Comparative Physiology. A: Sensory Neural and Behavioral Physiology, 180, 567-572.

Traugott, E., \& Dasher, R. (2005). Regularity in semantic change. Cambridge, UK: Cambridge University Press. 